\title{
Spatial Kerr beam self-cleaning in Yb-doped multimode fiber taper
}

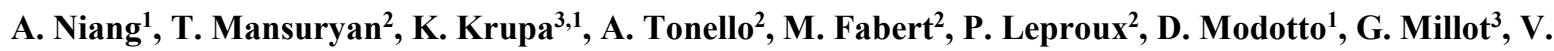 \\ Couderc $^{2}$, S. Wabnitz ${ }^{4}$ \\ 1. Dipartimento di Ingegneria dell'Informazione, Università degli Studi di Brescia, via Branze 38, 25123, Brescia, Italy \\ 2. Université de Limoges, XLIM, UMR CNRS 7252, 123 Avenue A. Thomas, 87060 Limoges, France \\ 3. Université Bourgogne Franche-Comté, ICB, UMR CNRS 6303, 9 Avenue A. Savary, 21078 Dijon, France \\ 4. DIET, Sapienza Università di Roma, via Eudossiana 18, 00184 Rome, Italy
}

Spatial Kerr beam self-cleaning (KBSC), which transforms an output speckled beam in a quasi-single mode beam in graded index (GRIN) multimode optical fibers (MMFs), has been reported recently [1,2]. GRIN MMFs are also interesting waveguides for supercontinuum (SC) generation in the visible and near infrared regions, as successfully demonstrated by launching femtosecond or subnanosecond pulses in the anomalous (1550 nm) or normal $(1064 \mathrm{~nm})$ dispersion regime, respectively [1,3]. The spectral broadening features were explained through the interplay between spatiotemporal multimode soliton oscillations and dispersive wave generation in fibers with parabolic index profile. On the other hand, tapered optical fibers are well known to provide a number of useful features, including strong mode confinement capability, small scale diameter and broad SC generation [4,5]. Herein, we report visible-short infrared SC generation in combination with KBSC in long ( 10 m) tapered Ybdoped MMFs, with parabolic index profile and parabolic doping concentration.

In our experiment we used a $10 \mathrm{~m}$ long Yb-doped MMF exhibiting quasi-parabolic core refractive index profile (see fig.1c). The largest input face of the taper was $122 \mu \mathrm{m}$, whereas the smaller one was close to $40 \mu \mathrm{m}$. Our taper was first excited with a $1064 \mathrm{~nm}$ laser source emitting $500 \mathrm{ps}$ pulses with more than $130 \mathrm{~kW}$ of peak power. A CW multimode laser diode (LD) at $940 \mathrm{~nm}$ was also used, in order to pump the rare-earth $\mathrm{Yb}$ ions, which provide gain along the propagation in the tapered fiber. In a first experiment, we observed KBSC in a passive configuration, i.e., switching off the $\mathrm{CW}$ pump diode. When increasing the power of the laser source, the spatial beam pattern at the fiber output evolved significantly, from a speckled beam into a quasi-single mode emission, matching well the fundamental mode profile of the fiber (see fig.1d). Because of the residual absorption of the fiber at $1064 \mathrm{~nm}$, the residual output power is to $9.35 \mathrm{~kW}$ (input power: $110 \mathrm{~kW}$ ). In these conditions, no significant frequency conversion was obtained, except for the first Raman Stokes sideband.

In a second step, we added the pump at $940 \mathrm{~nm}$ for amplification along the multimode fiber taper. Under this configuration, the input signal wave was amplified mainly over the first meter of propagation. Additionally, by further increasing the CW pump at $940 \mathrm{~nm}$, we obtained a high-beam quality SC emission between $520 \mathrm{~nm}$ and $2600 \mathrm{~nm}$ (see fig 1 (e)). The visible part of the spectrum is obtained thanks to parametric processes, whereas the infrared spectrum is mainly built-up by soliton propagation and Raman self-frequency shift.

(a)

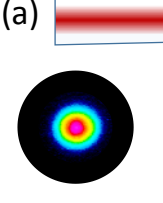

Input beam

(b)
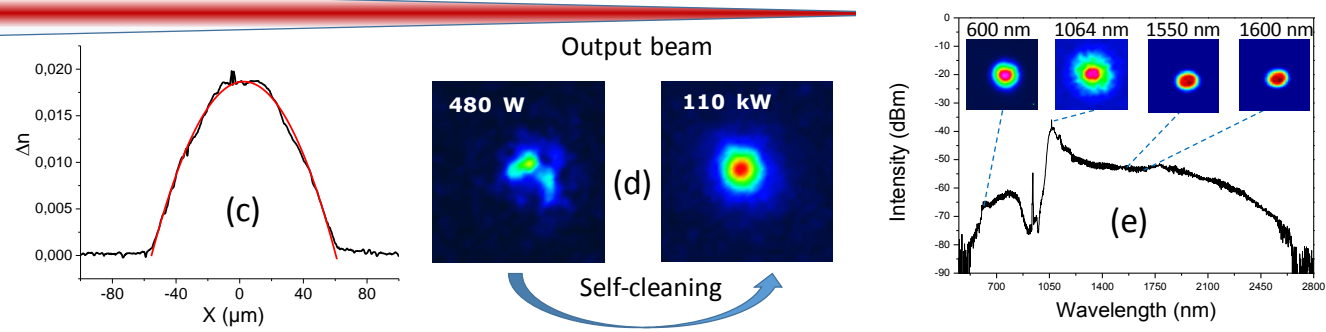

Fig. 1: (a) Doped multimode fiber taper, (b) input beam shape at $1064 \mathrm{~nm}$, (c) core refractive index profile, (d) output beam shape for two input peak powers at $1064 \mathrm{~nm}$ without $\mathrm{CW}$ pump (left: linear propagation, right: nonlinear propagation and spatial self-cleaning), (e) experimental spectrum obtained in the active taper with $8.3 \mathrm{~kW}$ input power at $1064 \mathrm{~nm}$ and maximum gain value of 3 , inset: near-field output beam profile with different bandpass filters.

\section{References}

[1] L.G. Wright, D.N. Christodoulides, F.W. Wise, "Controllable spatiotemporal nonlinear effects in multimode fibres,” Nat. Photonics 9, 306 (2015).

[2] K. Krupa, A. Tonello, B.M. Shalaby, M. Fabert, A. Barthélémy, G. Millot, S. Wabnitz, and V. Couderc, "Spatial beam self-cleaning in multimode fiber," Nat. Photonics 11, 237-241 (2017).

[3] G. Lopez-Galmiche, Z. S. Eznaveh, M. A. Eftekhar, J.A. Lopez, L.G. Wright, F. Wise, D. Christodoulides, R.A. Correa, "Visible supercontinuum generation in a graded index multimode fiber pumped at $1064 \mathrm{~nm}$," Opt. Lett. 41, 2553-2556 (2016).

[4] T. Birks, W. J. Wadsworth, and P.S.J. Russell, "Supercontinuum generation in tapered fibers," Opt. Lett. 25, 1415 (2000).

[5] M. A. Eftekhar, Z. Sanjabi-Eznaveh, J. E. Antonio-Lopez, H. Aviles, S. Benis, M. Kolesik, A. Schülzgen, F. W. Wise, R. Correa, D. N. Christodoulides, "Accelerating nonlinear interactions in tapered multimode fibers," in Conference on Lasers and Electro-Optics, Optical Society of America, (2018). 